\title{
An optimized time screening algorithm for ROSAT PSPC and HRI observations
}

\author{
F. Bocchino, M. Barbera, and S. Sciortino \\ Istituto ed Osservatorio Astronomico "G.S. Vaiana", Piazza del Parlamento 1, I-90134 Palermo, Italy \\ e-mail: bocchino@oapa.astropa.unipa.it, barbera@oapa.astropa.unipa.it, \\ sciorti@oapa.astropa.unipa.it
}

Received June 5; accepted October 20, 1997

\begin{abstract}
We have developed a model-independent time screening optimization algorithm to cope with significant contamination spikes in the ROSAT PSPC/HRI bacground light-curves. The rejection criteria are based on the maximization of faint sources signal-to-noise ratio. The algorithm tuning parameters have been optimized through performing a wide set of runs on both simulated and real data. We have verified that the application of our selection criteria to the case of long exposure PSPC observations yields an increase of the number of faint sources $(S N R<6 \sigma)$ of up to $100 \%$ with a rejection of up to the $8 \%$ of the exposure time. At the same time, we obtain an average signal-to-noise ratio gain of $3 \%$ for the sources detected in both the unscreened and screened images. The algorithm, even though currently optimized for the instrumentation on board ROSAT, can be easily generalized for data analysis of other imaging X-ray detectors.
\end{abstract}

Key words: methods: data analysis - techniques: miscellaneous

\section{Introduction}

During a typical ROSAT observation, a number of spurious events are recognized as X-rays by the acquisition system. A classification scheme for this kind of event includes particle background (PB), scattered solar X-ray background (SB) and internal noise (IN). In the case of the ROSAT Position Sensitive Proportional Counter (PSPC, Pfeffermann \& Briel 1986), a model exists for the contribution of PB and SB (Snowden et al. 1992, S92 hereafter; Snowden \& Freyberg 1993, SF93 hereafter), while the internal noise is negligible. In the case of the HRI, a model exists for the IN (David et al. 1995) and PB (Snowden,

Send offprint requests to: F. Bocchino in preparation), but the effect of SB has not been investigated in detail as for the PSPC case. In all cases, the standard procedure to reduce the effects of the contamination by non cosmic backgrounds involves, at a certain point, a "cut" of putative offending observation intervals. The choice of what and where to cut is crucial, since it should actually be an optimal trade-off between two effects: the decrease of science target counting statistics and the reduction of the contamination by spurious events.

Here we describe a procedure to screen out the high background intervals from X-ray observations done with the ROSAT PSPC or HRI. Our approach differs from the one in S92 and SF93, because of the following reasons. First, it is model independent, i.e. it does not try to model the origin of the background, but it operates on the observed shape of the background light curve, thus eliminating the dependency of the method on the input model parameters as well as on the validity of the model itself. Second, it is optimized in order to guarantee that the resulting screening maximizes the signal-to-noise ratio $(S N R)$ of faint point-like sources in the field of view (FOV). Since detection algorithms operate typically at a fixed source acceptance probability threshold, i.e. at a given $S N R$, the application of the optimized screening procedure may yield a significant increase of the number of faint sources detected in the FOV.

Our paper is organized as follows: in Sect. 2 we describe the screening algorithm from a general point of view. In Sects. 3 and 4, we apply the formalism developed in Sect. 2 to the ROSAT PSPC and HRI cases and we draw some preliminary conclusions. In Sect. 5, we outline the operative recipe of the PSPC screening code we have developed under the IRAF environment, and in Sect. 6 we show the results of the code in the case of a PSPC image. Finally, in Sect. 7, we summarize our results. 


\section{Description of the screening procedure}

The aim of the calculation described here is to derive a quantity to be maximized in order to obtain the optimal screening. This quantity (hereafter the efficiency function) is defined as the ratio of a source signal to noise ratio computed in a time screened observation $\left(S N R_{\mathrm{S}}\right)$ and in the entire unscreened observation $\left(S N R_{\mathrm{U}}\right)$, as a function of the amount of rejected exposure time. We define a circular source cell and an annular background cell, whose sizes are related to the local PSF. The following quantities refer to the unscreened observation:

$$
\begin{aligned}
& C=\text { total counts in the detection cell (measured) } \\
& T=\text { total counts in the background cell (measured) } \\
& S=\text { source counts } 1 \\
& B=\text { background counts in the detection cell } \\
& \alpha=\text { fraction of source counts in the detection cell } \\
& \beta=\text { fraction of source counts in the background cell } \\
& r=\text { ratio of the background to detection cell area. }
\end{aligned}
$$

We can thus write, following the recipe of Harnden et al. (1984) and Kashyap et al. (1994):

$C=\alpha S+B$

$T=\beta S+r B$.

With simple algebra we can obtain from Eqs. (1) and (2) the following expression for the source counts:

$S=\frac{r C-T}{\alpha r-\beta}$.

Adopting Poissonian statistics on the measured quantities $C$ and $T$ we can also write the standard deviation on the source counts as:

$\sigma_{\mathrm{S}}=\frac{1}{\alpha r-\beta} \sqrt{T+r^{2} C}$.

Using the above equations, we can now write the signal to noise ratio of the source for the unscreened observation in terms of $S$ and $B$ :

$S N R_{\mathrm{U}}=\frac{S}{\sigma_{\mathrm{S}}}=\frac{S(r \alpha-\beta)}{\sqrt{S\left(\beta+r^{2} \alpha\right)+B\left(r+r^{2}\right)}}$.

If a fraction $x$ of the total observation time is screened out, the expressions for screened $C$ and $T$ becomes:

$C_{\mathrm{S}}=(1-x) \alpha S+B(x)$

$T_{\mathrm{S}}=(1-x) \beta S+r B(x)$

where $B(x)$, the total background present in the screened observation, is equal to $B$ (Eqs. (1) and (2)) for $x$ equal

\footnotetext{
$1 \quad S$ refers to the "true" source counts and not to the source counts in the detection cell.
}

zero (unscreened observation). The source is assumed to be constant over the observation.

Analogously to what we have done for the unscreened observation we can derive the screened $S N R$ :

$S N R_{\mathrm{S}}=\frac{r C_{\mathrm{S}}-T_{\mathrm{S}}}{\sqrt{T_{\mathrm{S}}+r^{2} C_{\mathrm{S}}}}$.

Using Eqs. (6) and (7), this becomes:

$$
S N R_{\mathrm{S}}=\frac{S(1-x)(r \alpha-\beta)}{\sqrt{S(1-x)\left(\beta+r^{2} \alpha\right)+B(x)\left(r+r^{2}\right)}} \cdot
$$

We can now define the efficiency function $E(x, r, \alpha, \beta, S, B)=\frac{S N R_{\mathrm{S}}}{S N R_{\mathrm{U}}}$ to be maximized in order to obtain the optimal screening of the observation under analysis.

$$
\begin{aligned}
& E(x, r, \alpha, \beta, S, B)= \\
& \frac{(1-x) \sqrt{S\left(\beta+r^{2} \alpha\right)+B\left(r+r^{2}\right)}}{\sqrt{S(1-x)\left(\beta+r^{2} \alpha\right)+B(x)\left(r+r^{2}\right)}} .
\end{aligned}
$$

For further discussions it is useful to rewrite the above equation to stress the dependence of the efficiency function on the ratios $B / S$ :

$$
E(x, r, \alpha, \beta, S, B)=\frac{(1-x) \sqrt{\left(\beta+r^{2} \alpha\right)+\frac{B}{S}\left(r+r^{2}\right)}}{\sqrt{(1-x)\left(\beta+r^{2} \alpha\right)+\frac{B(x)}{S}\left(r+r^{2}\right)}} \cdot
$$

The parameters $r, \alpha$, and $\beta$ depend on the geometry of the source and background cells and can be tabulated once the PSF of the instrument is known as a function of the off-axis angle at a given energy (or for a given input source spectrum). We point out that this formalism is very general with respect to the choice of the geometry of the source and background cells, and, in addition, it is independent from the shape of the instrumental PSF.

\section{The PSPC case}

The algorithm described can be straightforwardly applied for screening ROSAT PSPC observations. Two major background anomalies that may cause a decrease in signal to noise ratio of sources can be identified in the background light curve of a ROSAT PSPC, namely: 1) high particle background as identified by high master veto count-rate, and 2) times of high solar background and other short-term enhancements. We will separately discuss the effect on the light curve of each of them, and we will show that, while the $\mathrm{PB}$ has to be screened by an "ad-hoc" cut, the SB can be effectively reduced by the procedure described in Sect. 2.

\subsection{High particle background}

During a typical ROSAT PSPC observation, several kinds of charged particles, including cosmic rays, hit the 

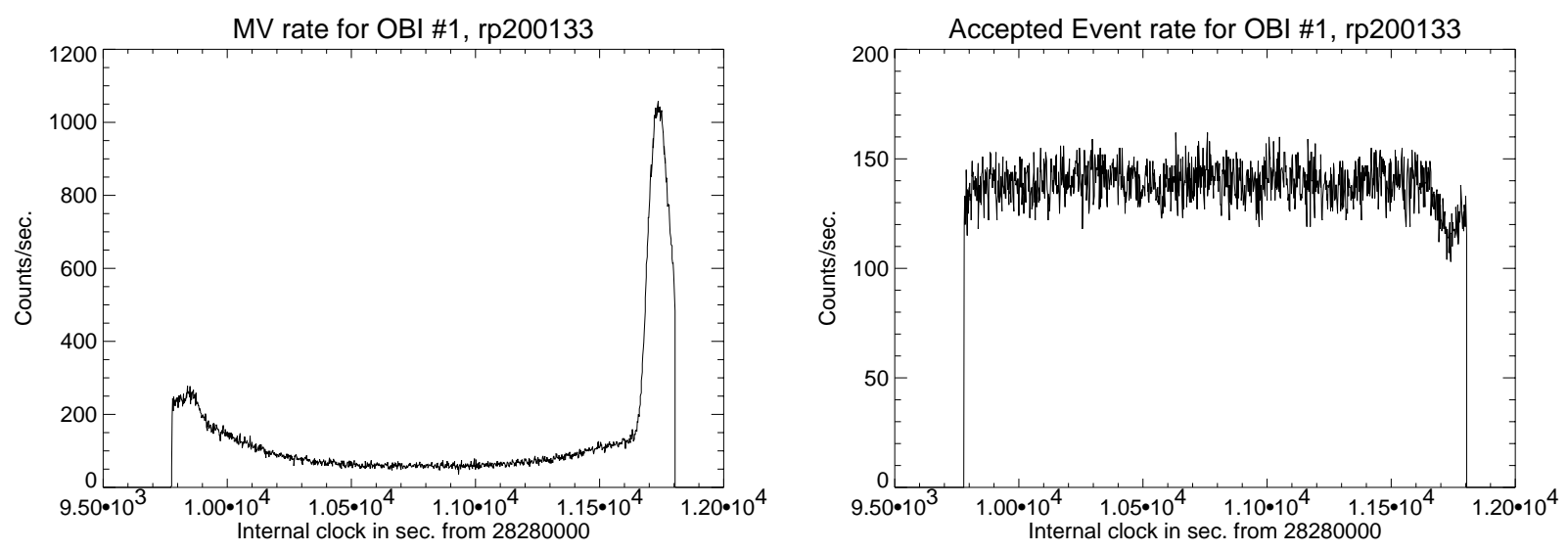

Fig. 1. Left: Master Veto (MV) light curve of the first Observation Interval (OBI) of the ROSAT PSPC sequence RP 200133 pointed toward the NE rim of the Vela supernova remnant (3944 sec total exposure). On average the MV rate is $<250 \mathrm{cnt} \mathrm{s}{ }^{-1}$, but at the end of the OBI the satellite has entered an high particle background region which increases the rejection rate above $1000 \mathrm{cnt} \mathrm{s}^{-1}$. Right: Accepted X-ray events (AE) rate for the same OBI as before. There is an apparent decrease of the rate corresponding to the high particle background interval of the left panel, because the high rejection rate increases the detector dead time. The dip is not real also because no source intrinsic variability is expected in the case of the rim of the Vela supernova remnant on the time scale of tens of seconds

detector, and cause electron cascades which are afterward marked as X-ray events by the read-out electronics. The PSPC has been equipped with an anti-coincidence counter, which detects, logs, and rejects these particle events. The rate of the rejected events is called the Master Veto (MV) rate. Nominally, $\gtrsim 99.8 \%$ of particle events are rejected. S92 have shown that, for typical MV values (MV $\lesssim 170$ cnt s$^{-1}$ ), the residual particle background (that is the particle events which are not rejected by the anticoincidence method) is negligible with respect to other background components (e.g. cosmic).

Under some circumstances, the satellite enters regions of high particle background (HPB) during an observing interval, signalled by a sudden increase of the MV rate. In these cases, the residual particle background may become comparable to other background rates (e.g. cosmic). To reduce the contamination of HPB, S92 suggested the screening out of time intervals where the MV rate is $>170 \mathrm{cnt} \mathrm{s}^{-1}$. This choice rejects $\sim 10 \%$ of the exposure time. We have verified that rejecting such an high fraction of the time may yield a net loss of the number of point-like sources detected in the screened observation and a systematic decrease of their $S N R$ 's.

On the other hand, Figs. 6 and 8 of S92 show that, for a MV rate up to $400 \mathrm{cnt} \mathrm{s}{ }^{-1}$, the residual particle background is still lower than $2 \mathrm{cnt} \mathrm{s}^{-1}$ (40\% and $15 \%$ of the minimum and typical X-ray cosmic background, respectively), and that choosing the MV threshold $\gtrsim 200 \mathrm{cnt} \mathrm{s}^{-1}$ yields a significant reduction of the discarded time intervals. However, as the MV rate increases, the detector dead time also increases. This is clearly shown in Fig. 1, which reports the MV light curve (left panel) of a PSPC observation pointed to the NE rim of the Vela supernova remnant (Bocchino et al. 1994) along with the accepted event rate of the same time interval (right panel). We note that, towards the end of the exposure, the MV rate increases significantly; when it becomes larger than about $250 \mathrm{cnt} \mathrm{s}^{-1}$, the accepted event rate shows an apparent drop due to high detector dead time. This evidence suggested, as a first step of our PSPC screening procedure, to reject the time intervals with $\mathrm{MV}$ rate $>250 \mathrm{cnt} \mathrm{s}^{-1}$. This choice is not restrictive in terms of exposure times (according to S92, only less than $1 \%$ of the exposure is rejected when only intervals with $\mathrm{MV}$ rate $<250 \mathrm{cnt} \mathrm{s}^{-1}$ are retained), and allows us to reject observation intervals with critical dead time. Adopting this MV threshold, we can estimate, on the basis of the typical and minimum rate of the cosmic X-ray background and Fig. 8 of S92, that the $\mathrm{PB}$ contribution to the total background is always $<25 \%$, with a typical value of $<5 \%$.

\subsection{Short term enhancement}

A typical background light-curve of a ROSAT PSPC or HRI observation is characterized by occasional steep enhancements in the total accepted event count-rate. Some of these excess background intervals are due to solar radiation scattered by Earth's atmosphere and others have been identified as auroral X-rays. In Fig. 2 (left panel), for instance, we show a background light curve of a ROSAT/PSPC observation which suffers of several contaminating spikes. According to SF93, the spikes are due to scattered solar X-ray radiation which enters the mirror assembly when the pointing direction crosses the sunlit limb of Earth. 

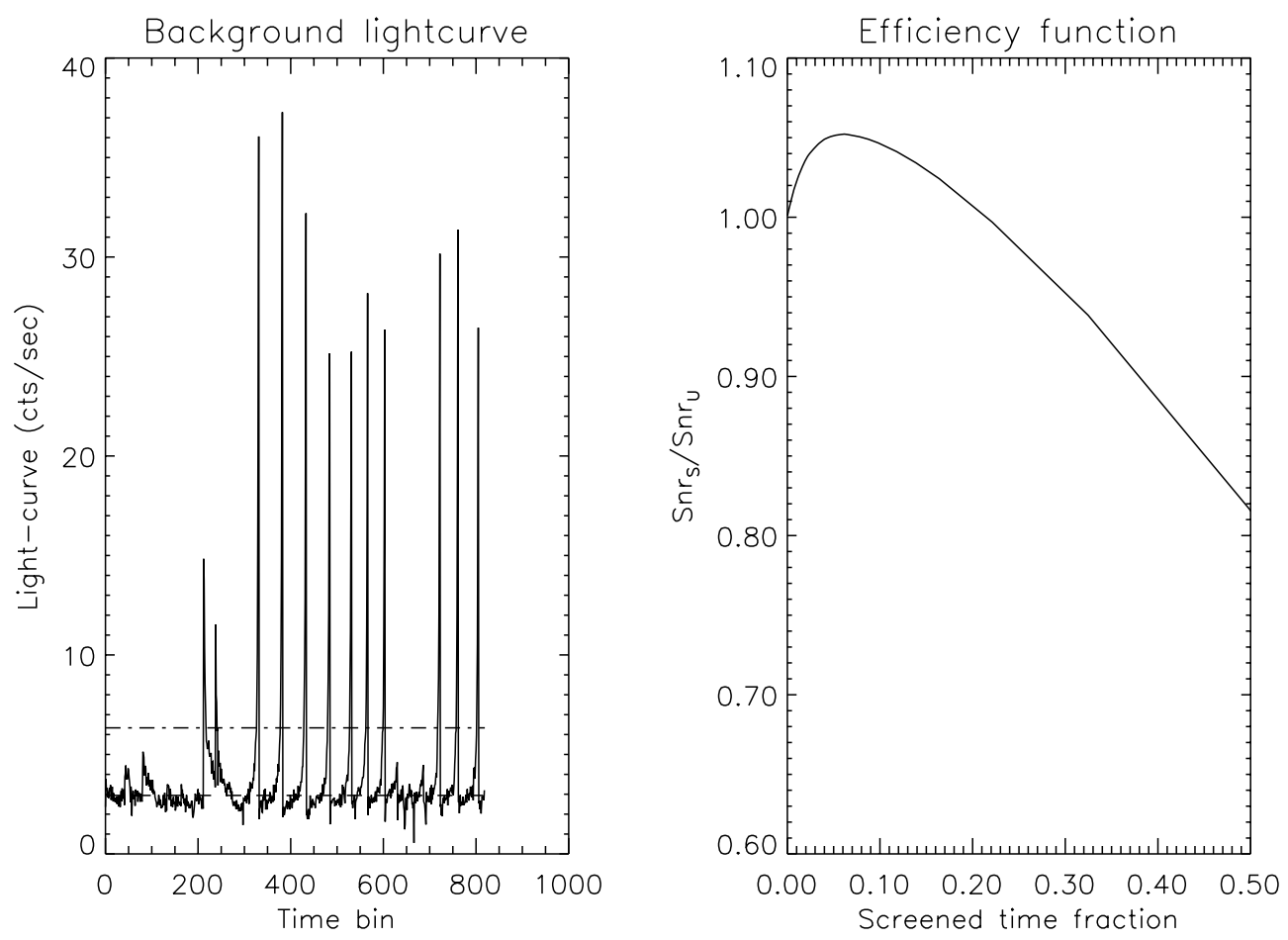

Fig. 2. Left: Background light curve of a PSPC field pointed towards the Pleiades open cluster. A large number of steep background enhancements are present along the entire $\sim 40000$ s observation interval. Dashed line marks the median background level, dot dashed line is the threshold level chosen by the screening algorithm described in the text to operate the rejection of the contaminating spikes. Right: The Efficiency Function defined in the text versus the fraction of the total exposure time screened out. The maximum of the curve is automatically selected by the algorithm and corresponds to the cut marked by the dot dashed line in the left panel

Our screening algorithm is capable of removing these high background intervals. To use Eq. (11) in the PSPC case, we have chosen a circular source region which includes $90 \%$ of the source photons $(\alpha=0.9)$ and an annular background region whose inner radius is equal to the source region radius and whose area is three times the source region area $(r=3)$. From the integration of the PSPC point spread function (PSF) at $0.3 \mathrm{keV}$ given by Hasinger et al (1993), we found for $\beta$ a typical value of 0.08 for off-axis angles in the range $0^{\prime}-50^{\prime}$. With these choices $\alpha, \beta$, and $r$ are almost independent on off-axis position and the only relevant parameters remain $S$, and $B^{2}$.

\footnotetext{
2 We note, in passing, that the choice of an annular background region instead of circular regions used by Kashyap et al. (1994) can be justified with the following reasoning. From Eq. (5), we see that if $r=3$ and $\beta=\beta^{\prime}-\alpha$ (this is valid in case of background annulus, and $\beta^{\prime}$ is the integral of the PSF between 0 and the background outer radius), then $S N R_{\mathrm{U}}=S\left(4 \alpha-\beta^{\prime}\right) / \sqrt{S\left(\beta^{\prime}+8 \alpha\right)+12 B}$. If $r=4$ and $\beta=\beta^{\prime}$ (in case of background circle centered on the source), then $S N R_{\mathrm{U}}=S\left(4 \alpha-\beta^{\prime}\right) / \sqrt{S\left(\beta^{\prime}+16 \alpha\right)+20 B}$. Therefore the $S N R$ of a source in the latter case is lower.
}

\subsection{Test simulations}

Before we describe operatively the screening procedure, we discuss, on the basis of results of the simulation tests, the dependence of our screening procedure on the choice of the parameters on which the screening procedure is tailored (i.e. off-axis angle and count-rate of the point source of which the efficiency function is to be maximized). For these purposes we have generated a simulated PSPC image with exposure time and background light curve similar to a PSPC field of the Pleiades young open cluster with nearly $40000 \mathrm{~s}$ total exposure time (rp200068, PI R. Rosner) presenting many steep background enhancements (Fig. 2). We have conducted extensive tests on the simulated Pleiades PSPC field under study, running the screening procedure tailored to sources of different off-axis angles (hereafter, the template sources). For each run, a template source was generated with a number of counts between the detection threshold and 10 times the threshold. $C, T, S$ and $B$ were computed accordingly, and the optimal screening was found by maximizing the efficiency function (Eq. 11). This approach has allowed us to study the behavior of the algorithm by varying the characteristic of the template source. 
The tests show that when the screening is tailored to fainter template sources, more time is screened out to obtain the maximum $S N R$. This means that, when the screening procedure is tailored to obtain maximum $S N R$ for faint sources, more time intervals are screened out than necessary for obtaining maximum $S N R$ for brighter sources. Nevertheless a screening procedure tailored to faint sources usually produces also an increase in $S N R$ for bright sources, although not the maximum obtainable for these latter sources.

Once it is clear that it is advantageous to tailor the screening procedure to the faintest source that can be detected in the field under study (i.e. $S$ is equal to the detection threshold for a given image), we have to investigate whether the procedure is sensitive to the choice of the off-axis angle of the template source. In fact, as we move outside from the center of the field of view, the width of the PSF of the detector increases, and the detection cell needs to be increased in order to keep the fraction of source counts falling within it constant. This implies an increase of the background counts $B$, whose collection cell scales with the area of the detection cell. Furthermore, the larger the off-axis angle, the higher the detection threshold (due to PSF widening and vignetting effect); therefore, the behavior of $S / B$ in the efficiency function $E$ to be maximized is not directly obvious.

Table 1 summarizes the results of these tests. Column 1 is the off-axis angle; Col. 2 gives the associated detection cell radius; Col. 3 is the detection threshold for that off-axis angle derived using the Wavelet transform detection algorithm of Damiani et al. (1997a); Col. 4 gives the amount of screened time $(x)$; Col. 5 gives the ratio $S N R_{\mathrm{S}} / S N R_{\mathrm{U}}$ i.e. the $S N R$ gain for the source to which the screening is tailored; Cols. 6, 7, and 8 give the $S N R$ gain which would be obtained for the source if the procedure had been tailored to $10^{\prime}, 20^{\prime}$ or $30^{\prime}$ off-axis angles. This table shows that the choice of tailoring the algorithm to large off-axis angles does not affect the gain in $S N R$ of sources at smaller off-axis angles. For instance, an on-axis source (first row in in Table 1) would have a gain of $2 \%$ in $S N R$ with the choice of tailoring the algorithm to $30^{\prime}$ off-axis angle (Col. 8), which is the same gain which would be obtained tailoring the algorithm to the on-axis source (Col. 5). The table also shows that the optimal screening of sources at large off-axis angles is obtained tailoring the algorithm to template sources also at large off-axis angles. In fact, the last row of the table shows that the optimal screening for a $30^{\prime}$ off-axis source is obtained with a cut of $5.6 \%$ of the exposure time (Col. 4 ), while tailoring the algorithm at smaller off-axis angle would have yielded a smaller fraction of rejected time, and thus a $S N R$ gain for the $30^{\prime}$ source which is lower than the maximum achievable (Fig. 2).

However, it is evident that the screening algorithm is not very sensitive to the chosen off-axis angle. This indicates that the increase of the detection cell size, and thus
$B$, and the increase of the detection threshold that occurs at larger off-axis angles, in some way compensates, keeping $S / B$ nearly constant. On the basis of these results, we have chosen to tailor our screening procedure to an offaxis angle of $30^{\prime}$, where we have the highest gain in $S N R$ and we still have acceptable PSPC performances both in terms of sensitivity and spatial resolution.

\section{The HRI case}

Given its generality, the algorithm is portable to other detectors. In the case of the ROSAT HRI, we have used the same cell geometry, but different choices of $\alpha$ and $\beta$. We adopted the PSF given by David et al. (1995) and we have verified that the highest value of $\alpha$ usable for count extraction purposes is 0.85 . For greater $\alpha$, the calculation of the cell radius involves integration of the tails of the PSF rather than the core, and since the tail is less well known than the core, that would yield high uncertainties in the assessment of the cell's size.

We have performed simulations similar to those described in the PSPC case. We verified that the screening algorithm is more efficient when tailored on a template source lying at $15^{\prime}$ off-axis in the HRI FOV. Since the HRI FOV is $\sim 18^{\prime} \times 18^{\prime}$, this result is therefore similar to the PSPC case, in which the optimal template source lies at $30^{\prime}$ off-axis in a $\sim 55^{\prime}$ radius FOV.

We have also verified that the screening procedure yields very low gain on detected sources $(\lesssim 1 \%)$. The corresponding rejection percentages are also negligible, usually between 1 and $3 \%$ of the total exposure times. We stress that this result is not due to low background counting statistics in the detection cell, because the ratio between the number of source and background counts in the cell is $\sim 1 / 5$ in both PSPC and HRI typical observations. Other explanations of the low HRI screening efficiency could be:

1. The HRI background is dominated by the particle component. In particular, the peaks in the HRI background light-curve are due to high particle rate intervals. The peak amplitude, however, is mostly only a factor of 3 higher than the median background value, with few cases in which it is a factor of 10 higher (Fig. 3). Instead, in the PSPC case, the peaks are mostly a factor of 10 higher than the median, with few cases in which they are only a factor of two higher. Our algorithm, therefore, tends to exclude more time intervals in the PSPC case rather than in the HRI case, because the HRI background light-curves are relatively less contaminated by spikes than those of the PSPC.

2. The HRI spikes are broader than the PSPC ones, because they are due to the particle component which is just a function of the sub-satellite position and does not change as rapidly as the SB PSPC spikes.

We have carried out an additional set of simulations to investigate which of our proposed explanations are correct. We have simulated an HRI background light curve 
Table 1. Analysis of the dependence of the screening procedure on template source off-axis angle

\begin{tabular}{cccccccc}
\hline \hline $\begin{array}{c}\text { Off-axis } \\
(\operatorname{arcmin})\end{array}$ & $\begin{array}{c}\text { Cell Radius } \\
(\operatorname{arcmin})\end{array}$ & $\begin{array}{c}S \\
(\mathrm{cnt} / \mathrm{s})\end{array}$ & $\begin{array}{c}X \\
(\%)\end{array}$ & $\frac{S N R_{\mathrm{S}}}{S N R_{\mathrm{U}}}$ & $\left(\frac{S N R_{\mathrm{S}}}{S N R_{\mathrm{U}}}\right)_{10^{\prime}}$ & $\left(\frac{S N R_{\mathrm{S}}}{S N R_{\mathrm{U}}}\right)_{20^{\prime}}$ & $\left(\frac{S N R_{\mathrm{S}}}{S N R_{\mathrm{U}}}\right)_{30^{\prime}}$ \\
\hline 0 & 0.475 & $6.710^{-4}$ & 3.7 & 1.02 & 1.02 & 1.02 & 1.02 \\
2 & 0.492 & $6.810^{-4}$ & 3.9 & 1.02 & 1.02 & 1.02 & 1.02 \\
4 & 0.492 & $6.910^{-4}$ & 3.7 & 1.02 & 1.02 & 1.02 & 1.02 \\
6 & 0.542 & $7.510^{-4}$ & 3.9 & 1.03 & 1.02 & 1.02 & 1.02 \\
8 & 0.592 & $8.210^{-4}$ & 4.2 & 1.03 & 1.03 & 1.03 & 1.03 \\
10 & 0.692 & $9.310^{-4}$ & 4.5 & 1.03 & 1.03 & 1.03 & 1.03 \\
15 & 0.992 & $1.210^{-3}$ & 5.4 & 1.04 & & 1.04 & 1.04 \\
20 & 1.408 & $2.010^{-3}$ & 5.4 & 1.04 & & 1.04 & 1.04 \\
25 & 1.875 & $2.210^{-3}$ & 5.6 & 1.05 & & & 1.05 \\
30 & 2.391 & $2.910^{-3}$ & 5.6 & 1.05 & & & 1.05 \\
\hline
\end{tabular}
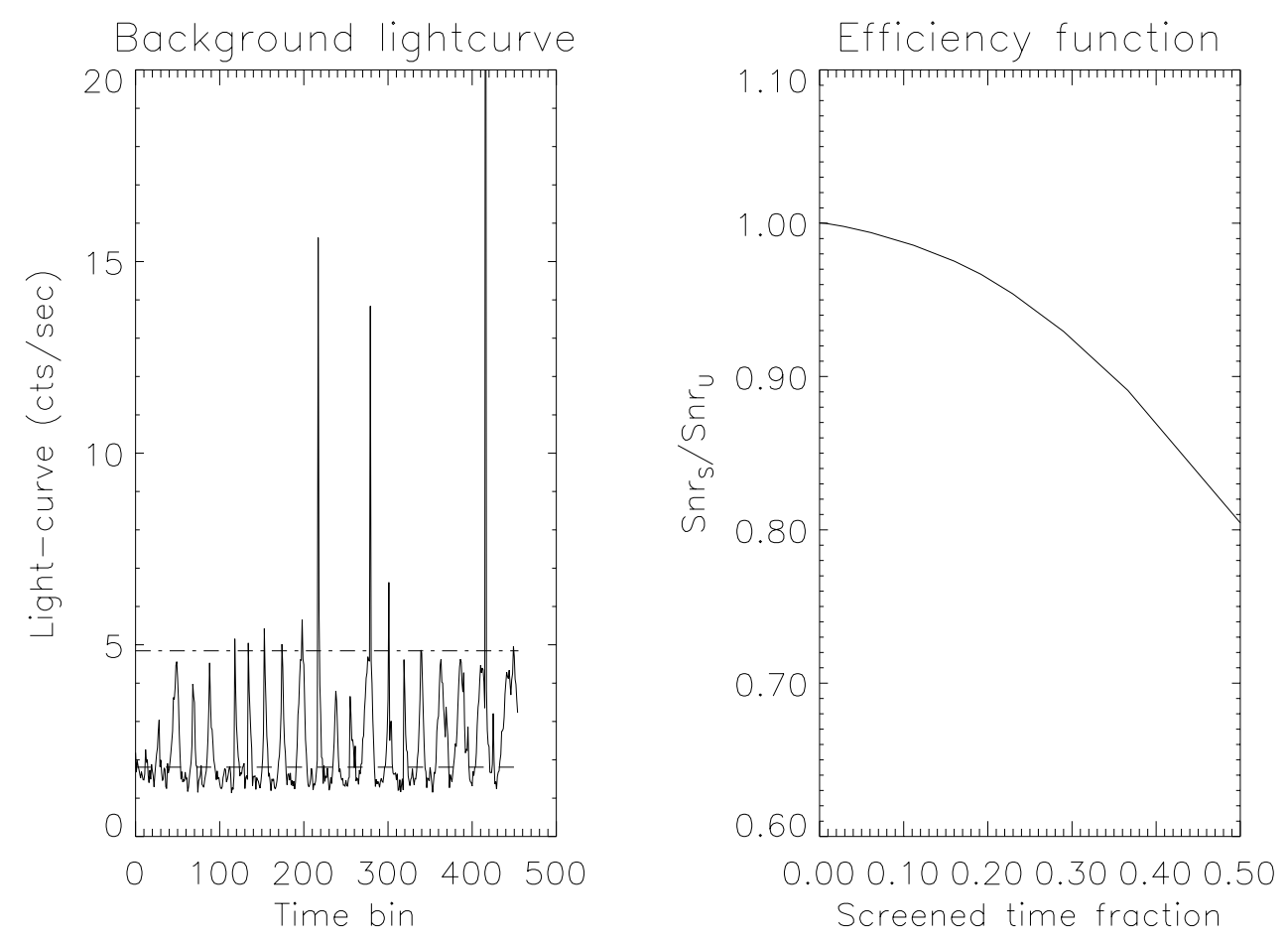

Fig. 3. Same as Fig. 2 but for the background light curve of an HRI field pointed towards the NGC 2422 open cluster (38075 s)

as a Gaussian spike superimposed on a constant level, and we run the screening procedure varying the Gaussian amplitude and sigma one at a time. The screening efficiency turned out to be rather insensitive to the width of the spikes, but very sensitive on the ratio between the Gaussian amplitude and the constant background level. In particular, the screening gain is very low when the ratio is comparable to that observed in typical HRI images, and it yields a not negligible gain $(>1 \%)$ when the ratio is at least twice as observed, as usually occurs in PSPC observations.

Figure 3, left panel, reports the light curve of an HRI case study, a sequence pointed toward the NGC 2422 open cluster with $\sim 70$ detected sources (RH 201828, Barbera et al. in preparation).

After inspecting a number of HRI real background light-curves, we conclude that the screening of HRI data is not a crucial point in HRI data analysis, but it could likely be very effective when the particle and/or the SB spikes heavily contaminates the data. This may occur either when the spike amplitudes are large (like in PSPC) or when the mean background level is low (this may be the case of the AXAF detectors). 


\section{The operative recipe}

Once we have identified the optimal parameters for maximum $S N R$ gain for faint point sources, we pass on to describe operatively the screening algorithm. From here on, we concentrate on PSPC data. Given a PSPC image, the application of our screening algorithm proceeds according to the following steps:

1. we build the MV rate light curve and reject observation intervals with $\mathrm{MV}$ rate $>250 \mathrm{cnt} \mathrm{s}^{-1}$ to screen out residual particle events as described in Sect. 3.1;

2 . we determine the background count collection region. This is accomplished computing the $20 \%$ and $80 \%$ percentiles of the count-rate image, and retaining in the collection region the pixels which fall within this range. With this choice, we discard putative sources and possible shadows (i.e. PSPC ribs);

3 . we build the background light-curve of the field summing up all pixel counts in the image region defined in step 2 , and scale it to the detection cell size at $30^{\prime}$ offaxis angle; then, we sum up the background light-curve to obtain the background counts $B$;

4. using the Wavelet trasmorm algorithm of Damiani et al. (1997a), we compute the detection threshold of our field at $30^{\prime}$ off-axis angle and use it as the source count-rate in the screening procedure; we then multiply this count-rate by the exposure time in order to get the source counts $S$.

At this stage we have all the information necessary to run the screening procedure. We define a variable that we call background moving threshold and set it initially to the maximum value of the background light-curve. Step by step, we decrease this variable by small amounts (say $1 \%$ of the initial value). At each step we evaluate $x$ as the fraction of exposure time where the background count-rate is in excess of the moving threshold, and also evaluate $B(x)$ subtracting from $B$ the background counts in the above "excess" background intervals. Figure 4 gives a pictorial view of the quantities calculated at each step. Given $x$ and $B(x)$, the efficiency function $E$ can be calculated. When this function reaches its maximum, the screening procedure is stopped and the time intervals to be screened out are identified.

\section{A case study, a crowded PSPC field}

Our algorithm yields very satisfactory results for long PSPC sequences (exposure time $\gtrsim 25 \mathrm{ksec}$ ), since in these cases the observations have high probability to be contaminated by particle and/or solar background. Figure 5 shows the results of the screening procedure on RP 200020, a sequence pointed at the Hyades open cluster with $40412 \mathrm{~s}$ exposure time. The sources have been detected using the Wavelet transform algorithm developed by Damiani et al. (1997a). In this run, our algorithm has rejected $\sim 3300 \mathrm{~s}$ ( $8 \%$ of the total exposure

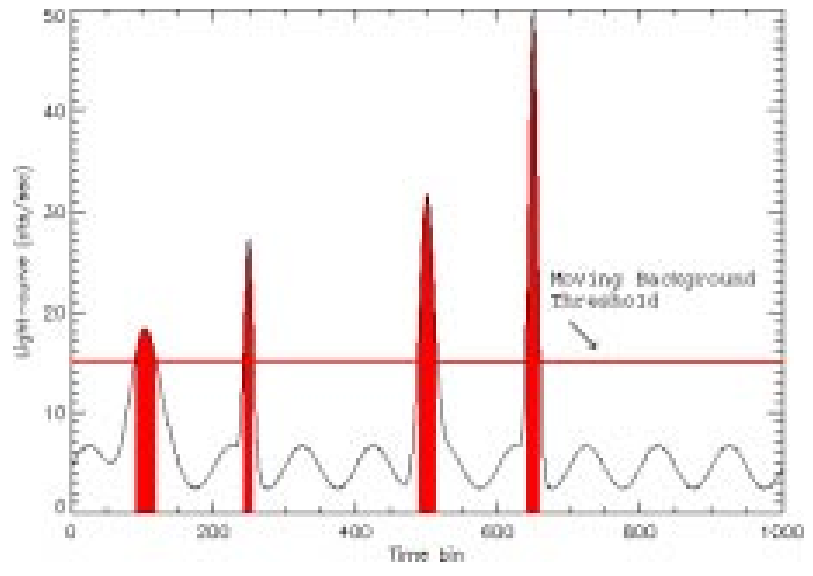

Fig. 4. In this simulated background light curve we put in evidence some of the relevant quantities involved in the screening procedure. The straight line is the background moving threshold. The shaded areas represent the intervals (and thus background counts) that are screened out at this stage of the procedure. $B(x)$ is the integral of the light curve out of the shaded areas

time). In the figure, we show a scatter plot of $S N R_{\mathrm{s}}$ ( $S N R$ of the sources in the screened observation) versus $S N R_{\mathrm{u}}$ ( $S N R$ of the sources in the unscreened observation). The detection threshold $(4.35 \sigma)$ is marked by the dashed lines ${ }^{3}$, while the solid line divides the plot in the region where $S N R_{\mathrm{s}}>S N R_{\mathrm{u}}$ (upper left) and the region where $S N R_{\mathrm{S}}<S N R_{\mathrm{u}}$ (lower right). We have displayed only the sources with $4<S N R<6$, to stress the behavior of the screening algorithm on faint sources. In the plot, we have 15 sources with $S N R_{\mathrm{s}}>S N R_{\mathrm{u}}$, of which 8 were not detected in the unscreened data but were detected in the screened data when running the detection algorithm at the $4.35 \sigma$ threshold. These sources are in the upper left shaded area. On the other hand, we have 9 sources with $S N R_{\mathrm{s}}<S N R_{\mathrm{u}}$, of which none moved below the detection threshold after the screening (lower right shaded area). We therefore have 8 new detections in the screened data set. Since there are 6 sources detected with $4.35<S N R<6$ in the unscreened sequence, we have obtained a gain of more than $100 \%$ in the number of faint detected sources by running our screening algorithm. In addition, we have an average $S N R$ gain of $\sim 4 \%$ for the matched sources with $4.35<S N R<6$ (2.5\% considering all sources with $S N R>4.35)$. This is in agreement with the results of previous tests on simulated data sets which showed the advantages of tailoring the algorithm for the case of a faint template source. We note that at the chosen

\footnotetext{
3 The $4.35 \sigma$ detection threshold corresponds to 1 spurious source in the whole PSPC FOV (Damiani et al. 1997b), and it is typically used when performing detections on PSPC pointed sequences. In order to study the effect of the screening procedure on the sources below the usually adopted detection threshold, we run the wavelet algorithm using the $4.0 \sigma$ threshold.
} 


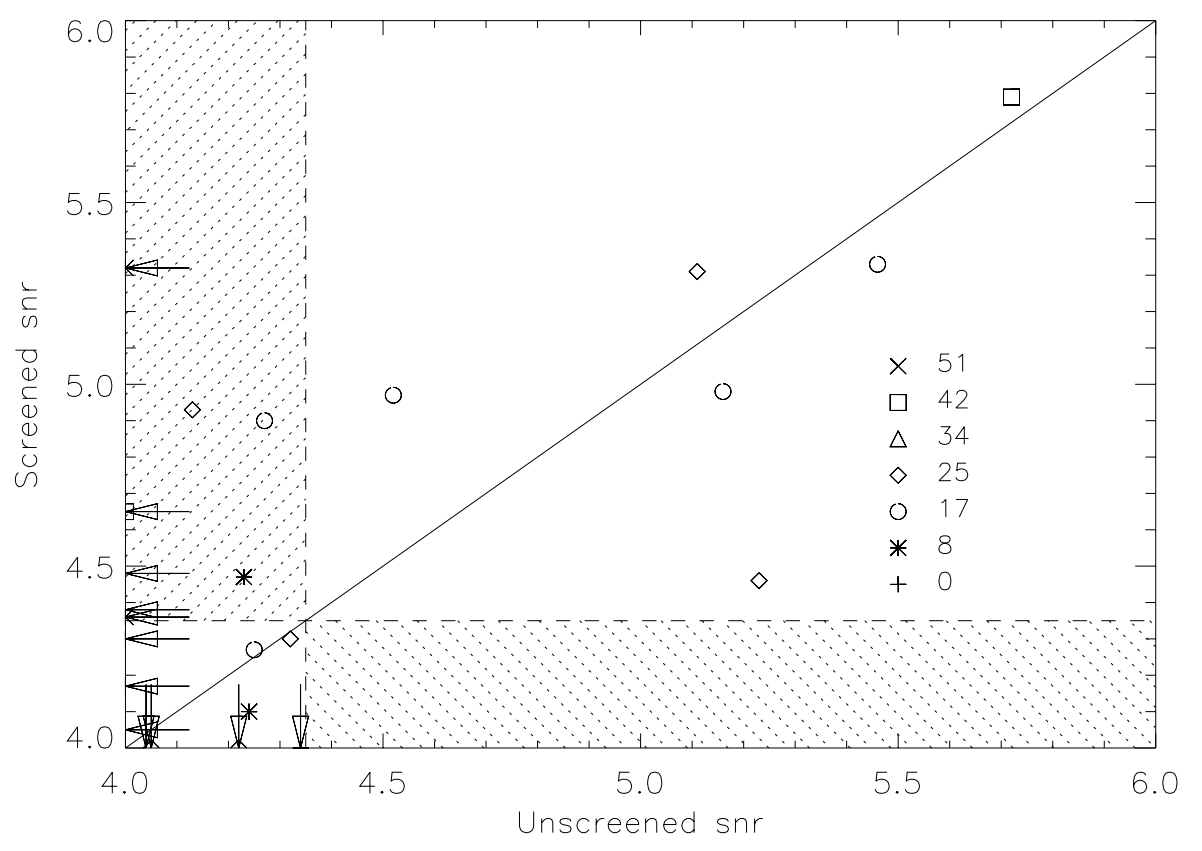

Fig. 5. $S N R_{\mathrm{u}}-S N R_{\mathrm{s}}$ scatter plot of RP 200020. Symbols represent off axis angles of displayed sources in arcmin (legend in the plot). Arrows mark upper limits for sources detected only, for instance, in the screened observation and not detected in the unscreened one or vice versa. Dashed lines correspond to the typical detection threshold used in the Wavelet transform detection algorithm of Damiani et al. (1997a,b), while the solid line marks $S N R_{\mathrm{u}}=S N R_{\mathrm{s}}$. Sources moved above detection threshold after the screening process lie in the upper-left dashed area with a dotted line pattern ( 8 new sources), while sources moved below the detection threshold would be in lower left dashed area (none in this case). New sources are not concentrated at large off-axis angles, where the PSPC PSF is not circular simmetric, but they are at a wide range of off-axis angles

threshold, the algorithm is expected to detect one spurious source per field (Damiani et al. 1997b), and therefore essentially all the new sources in the screened sequence should be real. To provide more confidence on this result we have tried to find counterparts for the 8 new sources using the SIMBAD database and the MPE ROSAT PSPC Catalogue, finding counterparts in 5 cases and none in the remaining.

We have verified that for short PSPC exposures ( $\lesssim 4 \mathrm{ksec}$ ), our algorithm tends to reject less than $\sim 1 \%$ of the total exposure time, in agreement with the expectations. In these cases, the average $S N R$ gain is very small, and the average number of gained sources is negligible.

\section{Summary and conclusions}

We have described a procedure developed to time-screen X-ray observations conducted with the ROSAT PSPC and HRI detectors which can be easily generalized to any photon counting detector with negligible internal background. The aim of this procedure is to maximize the $S N R$ of faint sources in the image, introducing a trade-off between cutting high background time intervals and keeping the observation interval the longest possible.
Test have been conducted in order to optimize the screening algorithm. It turns out that a convenient choice for the PSPC is to tailor the algorithm to the faintest detectable sources in the field at $30^{\prime}$ off-axis angle.

We have compared results of analysis on a PSPC moderately crowded field (pointed towards the Hyades open cluster) with no time-screening and time-screening applied according to our procedure. We have verified that a significant increment in the number of detected sources is obtained, as well as a gain in terms of the $S N R$ s of detected sources.

While the finding of new true sources is the most important result of our screening procedure, the systematic increase of detected sources $S N R$ is also important. In fact, since the detection algorithms operate at a fixed $S N R$, to which correspond a given number of expected spurious sources, the observed gain yields a decrease of contamination by fake sources, which is important especially in large area surveys.

Even though optimized for point sources, the screening procedure described here is applicable to automatic pipeline procedure, such as the one described in Mackie et al. (1996), since it does not fail in case of extended sources, and in particular when extended sources 
occupies a large fraction of the FOV. In fact, in these cases, our algorithm tend to reject a very small fraction of the total exposure time ( $\gtrsim 1 \%$ ) with respect to the case of sequences with point sources, because the extended source increases the background level and makes the $S N R$ ratio almost insensitive to the presence of contaminating spikes in the light curve.

Acknowledgements. The authors wish to thank the suggestion and comments from S. Serio. This work was partially supported by Ministero della Università e delle Ricerca Scientifica e Tecnologica, GNA-CNR and a contract of Italian Space Agency (ASI).

\section{References}

Bocchino F., Maggio A., Sciortino S., 1994, ApJ 437, 209

Damiani F., Maggio A., Micela G., Sciortino S., 1997a, ApJ 483,350
Damiani F., Maggio A., Micela G., Sciortino S., 1997b, ApJ 483,370

David L.P., Harnden Jr.F.R., Kearns K.E., Zombeck M.V., 1995, "The ROSAT High Resolution Imager (HRI), US. ROSAT Science Data Center/SAO"

Harnden F.R.Jr., Fabricant D.G., Harris D.E., Schwarz J., 1984, SAO Special Report 393

Hasinger G., Boese G., Predehl P., et al., 1993, MPE/OGIP Calibration Memo, CAL/ROS/93-015

Kashyap V., Micela G., Sciortino S., Harnden F.R.Jr., Rosner R., 1994, in "The Soft X-ray Cosmos", Schlegel E.M. (ed.) AIP Conf. Proc. 313, 239

Mackie G., Fabbiano G., Barbera M., et al., 1996, in proceeding of the Astronomical Data Analysis Software and Systems V, ASP Conf. Ser. (in press)

Pfeffermann E., Briel U.G., 1986, SPIE 597, 208

Snowden S.L., Freyberg M.J., 1993, ApJ 404, 403

Snowden S.L., Plucinsky P.P., Briel U., Hasinger G., Pfefferman E., 1992, ApJ 393, 819 